Azathioprine and its metabolite 6-mercaptopurine are effective in the treatment of inflammatory bowel disease. They are mostly used for reduction of the use of steroids, maintenance therapy after remission induction by cyclosporin and treatment of fistulae in Crohn's disease. Adverse effects occur in about $15 \%$ of patients. The main side effects are pancreatitis, allergic reactions, fever and bone marrow suppression. Symptoms, management and prevention are discussed. A blood monitoring schedule is suggested. Azathioprine and 6-mercaptopurine seem to be safe in pregnancy. There may be a slight increased $r$ isk for developing a non-Hodgkin's lymphoma.

Key words: azathioprine, 6-mercaptopurine, inflammatory bowel disease, bone marrow suppression, side effects, pregnancy

\section{Azathioprine in inflammatory bowel disease, a safe alternative?}

\author{
A. A. Tanis \\ Department of Gastroenterology and Hepatology, \\ Internal Medicine II, Erasmus University \\ Hospital-Dijkzigt, Dr. Molewaterplein 40, 3015 GD \\ Rotterdam, The Netherlands
}

Tel: $(+31) 104639222$

Fax: $(+31) 104633964$

Email:tanis@inw2.azr.nl

\section{Introduction}

Azathioprine (AZA) and its metabolite 6-mercaptopurine (6-MP) have been used in the treatment of inflammatory bowel disease (IBD) for more than 30 years. ${ }^{1}$ However the more widespread use of AZA/ 6-MP in the treatment of IBD is of more recent origin. The reason for this has been a longstanding debate on the efficacy of AZA/6-MP in IBD. Reasons for the doubt about efficacy were the relative long time before effects are seen, the different doses used, the different study designs and the small number of patients in some trials. AZA and 6-MP are slow acting drugs and the optimum effect may only be expected after 12-17 weeks of treatment.

In 1995 a meta-analysis of nine randomized, placebo-controlled clinical trials on the use of AZA/6-MP therapy in Crohn's disease was published. The estimated common odds ratio of response for active disease was 3.09 (95\% CI, 2.45-3.91) and for mainte nance therapy $2.27(95 \% \mathrm{CI}, 1.76-2.93)$. The steroid sparing effect had an odds ratio of $3.69(95 \% \mathrm{CI}$, 2.12-6.42) in active disease and 4.64 (95\% CI, $1.00-21.54)$ in quiescent disease. The improvement of fistulae with this therapy had an odds ratio of 4.44 (95\% CI, 1.50-13.20). In conclusion this analysis showed that AZA/6-MP is effective in active and quiescent Crohn's disease. ${ }^{2}$

This paper will deal with the safety of AZA/6-MP in the treatment of IBD. Adverse effects and questions relating to fertility and pregnancy are discussed. A monitoring schedule to minimize the risk of bone marrow suppression is suggested.

\section{Indications and Dosage}

The main indications for the use of AZA in IBD are (1) steroid sparing treatment, (2) maintenance of remission, (3) maintenance therapy after treatment with cyclosporin, (4) Crohn's disease with fistulae and (5) chronic active ulcerative colitis. Though Candy reported the successful use of AZA as a single drug treatment in remission induction and maintenance of remission of Crohn's disease, AZA is mostly used in combination with mesalasine or topical steroid therapy or both. ${ }^{3}$ The recommended dose for IBD is $1.5-2.5 \mathrm{mg} / \mathrm{kg}$ per day. ${ }^{2-4}$ Recently a high dose induction schemes using up $40 \mathrm{mg} \mathrm{AZA} / \mathrm{kg}$ over $36 \mathrm{~h}$ indicated that the time to response in Crohn's disease could be shortened. ${ }^{5}$ However such an induction scheme has the risk of serious side effects, especially in patients who lack the enzyme thiopurinemethyltranferase. This enzyme is responsible for converting one of its two inactive metabolites. A low or absent activity of this enzyme will result in higher concentrations of the active metabolites 6-thioguanine nucleotides (6-TGN). Larger studies are being performed to clarify the risk, benefit and safety of such a high dose induction scheme. ${ }^{6}$ Until such studies are completed the use of a high dose induction scheme should be limited to a study setting. Patients treated with this scheme should 
probably be tested for low thiopurinemethyltranferase activity prior to treatment.

\section{Adverse Effects}

Adverse effects serious enough to stop AZA/6-MP treatment may occur in $8-15 \%$ of IBD patients. ${ }^{2-4}$

The most common side effects of AZA/6-MP treatment are pancreatitis, allergic reactions, gastro-intestinal complaints, hepatitis and bone marrow suppression. There seems to be no difference in the type of side effects between AZA and 6-MP. It is therefore possible to use data from studies in which either one or both have been studied.

\section{Pancreatitis}

Of 396 IBD patients treated with 6-MP and followed up for a mean of 60.3 months, 13 patients $(3.3 \%)$ developed a clinical pancreatitis. ${ }^{4}$ The meta-analysis of Pearson et al. showed an overall occurrence of pancreatitis of $1.3 \% \%^{2}$ Others have reported pancreatitis to occur in $0-7 \%$ of patients. The mean duration between the start of the medication and the first symptoms the pancreatitis was 23 days, with a range of 1-32 days. How ever one patient developed a 6-MP related pancreatitis after 6 months of treatment. ${ }^{4,7}$ The clinical course of the pancreatitis is mild. When the AZA/6-MP medication is discontinued immediately when pancreatitis is diagnosed, symptoms disappear and serum amylase values return to normal within a mean of 3 days (range 1-11 days). No complications such as chronic pancreatitis or pseudocyst formation have been reported. No relation could be established between the pancreatitis and the dose of 6-MP or AZA, concurrent medication, other side effects such as bone marrow suppression or the severity of the IBD. ${ }^{2,4,7}$

Rechallenge with 6-MP or AZA after the patient had recovered from pancreatitis resulted in a relapse of the pancreatitis in seven of the seven cases. Desensibilization was tried in three patients without success. ${ }^{4}$

The usefulness of screening the amylase level in blood or urine has not been evaluated. However the rapid onset of the pancreatitis will make the chance of preventing clinical symptoms by early detection rather small. Secondly some patients develop a transient increase of the amylase level without clinical symptoms. The interpretation of screening data would therefore be difficult. This, taken together with the usual mild course and quick resolution of symptoms, suggests that laboratory tests for pancreatitis should only be performed on clinical indication.

\section{Allergic reactions}

Allergic reactions, including fever, skin rash and arthritis have been reported in up to $6 \%$ of IBD patients treated with AZA/6-MP. Patients usually become symptomatic within 3 weeks of treatment. Symptoms are mostly mild but can be severe. A hypersensitivity syndrome with multi-organ failure has occasionally been associated with the use of AZA. $^{2,4,8}$ Recovery occurs within a few days after discontinuation of the treatment. ${ }^{4}$ Rechallenge is not useful and could even induce a severe hypersensitivity syndrome. ' Successful desensitization for azathioprine skin rash in a patient with Crohn's disease has been reported recently. ${ }^{10}$

Two patients were recently described who developed a photosensitive rash while being treated with AZA for 10 and 14 days. The clinical diagnosis of pellagra was made. The dietary history showed a well balanced diet and an adequate nicotinic acid intake. The rash disappeared after nicotinic acid suppletion. 6-MP can cause a partial inhibition of the nicotinic acid pathway, leading to pellagra in patients who have already a marginal nicotinic acid status due to malabsorption caused by the IBD. ${ }^{11}$

\section{Hepatitis and gastro-intestinal complaints}

Toxic hepatitis due to AZA/6-MP is rare in patients being treated for IBD. Present et al. ${ }^{4}$ reported a patient who developed a toxic hepatitis on 6-MP, which resolved completely after withdrawal of the drug. A year later the patient rechallenged himself by restarting the drug to treat his colitis. The colitis responded well, but the hepatitis recurred within 6 weeks. Symptoms disappeared after withdrawal of the drug. ${ }^{4}$ Nausea, severe enough to stop treatment, has been reported in 1.3\% Mild symptoms of nausea or abdominal discomfort occur quite often after the start of the treatment with AZA/6-MP. The complaints are usually transient and dividing the dose into two or three a day may be helpful.

\section{Bone marrow suppression}

One of the most serious adverse effects of AZA/6-MP treatment in IBD is suppression of the bone marrow. All three cell lines can be affected. A severe leukopenia, thrombocytopenia or anaemia or a combination can be the result. Present reported a leukopenia of less then $2.5 \times 10^{9} / 1$ in $2 \%$ of the patients treated with $6-\mathrm{MP} 1.5 \mathrm{mg} / \mathrm{kg} /$ day. The meta-analysis of Pearson gave a mean of $1.7 \%$ One patient died due to septicaemia. ${ }^{2,4}$

Candy et al. ${ }^{3}$ reported a dose reduction of AZA due to a leukocyte count of less than $4 \times 10^{9} / 1$ in 13 of 33 patients. All returned to white cell count levels of above $4 \times 10^{9} / 1$ after dose reduction. No-one had to discontinue the treatment. ${ }^{3}$

A more detailed retrospective study was carried out in 739 patients, who were treated for IBD with an initial dose of AZA $2 \mathrm{mg} / \mathrm{kg} /$ day. The study covered a 
total of 1622 years of treatment. A leukopenia (white blood cell count less than $\left.3.0 \times 10^{9} / \mathrm{l}\right)$ and/or a thrombocytopenia (platelet count less than $100 \times$ $\left.10^{9} / \mathrm{l}\right)$ was recorded in 37 patients $(5 \%)$. Nine had a white cell count of less than $2.0 \times 10^{9} / 1$. Three of these nine patients were pancytopenic. Two of them died from septicaemia and the other had a pneumonia but recovered within a week. Another two patients with only severe leukopenia were symptomatic too. Both had a mild upper respiratory infection and recovered within a few days. Isolated thrombocytopenia was never clinically severe. Bone marrow toxicity from AZA/6-MP treatment may develop at any time. In the mentioned study the mean time at which the bone marrow suppression was diagnosed was 9 months with a range of $0.5-132$ months of treatment. Myelosuppression may occur suddenly or over a period of several months. ${ }^{1}$ No correlation was shown between bone marrow suppression and the cumulative dose of AZA. ${ }^{3,4}$ A negative correlation seems to exist between the white blood cell count and the effect of the treatment. ${ }^{3,4}$

Regular monitoring of the full blood count is recommended. However monitoring schedules differ in the different studies. The most practical and safe schedule seems to be a 2 -weekly monitoring for the first 3 months, followed by a bi-monthly monitoring schedule. The blood tests could be carried out at primary health care level, provided proper guidelines have been furnished. An example of a monitoring schedule is given in Table 1.

\section{Fertility and Pregnancy}

Many IBD patients are in the reproductive phase of their lives. This makes questions about the impact of AZA/6-MP treatment on fertility and pregnancy of great importance. The influence of AZA/6-MP on human fertility has not been studied. However there are indications that it does not cause infertility. ${ }^{12,13}$

The effect of the paternal use of AZA/6-MP at the time of conception on the outcome of the pregnancy is not entirely clear. The limited data available suggest it to be safe. There could be some concern from theoretical point of view and from some animal studies. However this is not supported by clinical evidence. ${ }^{13}$ Clinical experience with the use of AZA/ 6-MP during pregnancy is derived mostly from renal transplant recipients. In this group the use of AZA/ 6-MP during pregnancy may be associated with fetal growth retardation and prematurity. There seems to be no marked increase in the frequency of congenital anomalies or fetal death. ${ }^{13}$ The use of AZA/6-MP for IBD does not warrant a mandatory termination of pregnancy, as sometimes has been advised. ${ }^{14}$

It is advisable to discuss the small potential risks of the use of AZA/6-MP with the patient and the spouse before conception. In this discussion the indication
Table 1. Monitoring schedule azathioprine/6-mercaptopurine treatment for IBD

First 3 months: haemoglobin, leukocytes, platelets every 2 weeks, followed by Haemoglobin, leukocytes, platelets every 2 months

Haemoglobin $\downarrow 1-2 \mathrm{mmol} / /$ or
Leukocytes $3<-<4 \times 10^{9} / \mathrm{l}$ or
Platelets $70<-<100 \times 10^{9} / \mathrm{l}$

Action: Reduce dose to $50 \%$.

Check blood values weekly.

If further reduction, stop treatment and follow undermentioned scheme.

If values normalize, increase dose to $100 \%$.

Check blood values weekly for 1 month.

In case of a relapse, reduce dose to $50 \%$ permanently.

If no relapse, follow regular monitoring schedule.

Haemoglobin $\downarrow<2 \mathrm{mmol} / \mathrm{I}$ or
Leukocytes $<3 \times 10^{9} / \mathrm{I}$ or
Platelets $<70 \times 10^{9} / \mathrm{l}$

Action: Stop treatment.

Check blood values weekly.

If values normalize, restart with $50 \%$ of the initial dose.

Check blood values weekly for 1 month. In case of relapse, stop treatment permanently. If no relapse, follow regular monitoring schedule.

for which AZA/6-MP was prescribed and the risk of other drugs like high steroids and the risks of a flair up of the IBD should be pointed out as well.

\section{Neoplasms}

Are IBD patients, who are treated with AZA/6-MP for years, at greater risk to develop more neoplasms then the general population? This risk seems to be rather small. There is a possible association between AZA/ 6-MP use and the development of a (cerebral) lymphoma. If corrected for factors as age and underlying disease no other associations could be made. ${ }^{4,15,16}$

\section{Conclusions}

AZA/6-MP are effective in the treatment of IBD. They can be used as steroid sparing drugs, as maintenance drugs after remission induction with cyclosporin and for fistulae in Crohn's disease. The high dose intravenous induction treatment should only be used in a research setting until more data are available. The effective dose for $6-\mathrm{MP}$ is $1.5 \mathrm{mg} / \mathrm{kg} / \mathrm{day}$ and for AZA $1.5-2.5 \mathrm{mg} / \mathrm{kg} /$ day. The optimal effect is only reached after 3-4 months. The optimal length of treatment has still to be established. In about $15 \%$ of the patients the drug has to be discontinued because of adverse effects. The main adverse effects are pancreatitis, allergic reactions and bone marrow suppression. In pancreatitis or allergic reactions rechallenge seems to be of no use and could even be hazardous. To prevent 
severe bone marrow toxicity full blood count monitoring should be performed regularly. A schedule has been suggested in this paper. However clinicians should be alert to a sudden myelosuppression, which can occur despite regular monitoring.

When AZA/6-MP is the most effective drug for the treatment of IBD the re is not enough clinical evidence to advise against fathering a child or to become pregnant. Pros and cons should be discussed with the patient.

There may be a slight increased risk for developing a non-Hodgkin's lymphoma. The efficacy and safety of local AZA applications are presently being studied. This could be a promising development in the local treatment of IBD.

\section{References}

1. Connell WR, Kamm MA, Ritchie JK, Lennard-Jones JE. Bone marrow toxicity caused by azathioprine in inflammatory bowel disease: 27 years of experience. Gut 1993; 34: 1081-1085.

2. Pearson DC, May GR, Fick GH, Sutherland LR Azathioprine and 6-mercaptopurine in Crohn disease. A meta-analysis. Ann Intern Med 1995; 122: 132-142.

3. Candy S, Wright J, Gerber M, Adams G, Gerig M, Goodman R A controlled double blind study of azathioprine in the management of Crohn's disease. Gut 1995; 37: 674-678.

4. Present DH, Meltzer SJ, Krumholtz MP, Wolke A, Korelitz BI. 6-Mercaptopurine in the management of inflammatory bowel disease: short- and long-term toxicity. Ann Int Med 1989; 111: 641-649.

5. Sandborn WJ, van Os EC, Zins BJ, Tremaine WJ, Mays DC, Lipsky JJ. An intravenous loading dose of azathioprine decreases the time to response in patients with Crohn's disease. Gastroenterology 1995; 109: 1808-1817.
6. Sandborn WJ. 6-MP metabolite levels: a potential guide to Crohn's disease therapy. Gastroenterology 1997; 113: 690-692.

7. Haber CJ, Meltzer SJ, Present DH, Korelitz BI. Nature and course of pancreatitis by 6-mercaptopurine in the treatment of inflammatory bowel disease. Gastroenterology 1986; 91: 982-985.

8. Brown G, Boldt C, Webb JG, Halperin L Azathioprine-induced multi system organ failure and cardiogenic shock. Pharmacotherapy 1997; 17: 815-818.

9. Knowles SR, Gupta AK, Shear NH, Sauder D. Azathioprine hypersensitivity-like reactions-a case report and a review of the literature. Clin Exp Dermatology 1995; 20: 353-356.

10. Lavaud F, Abdelli N, Thiefin G. Successful desensitization for azathioprine skin rash in a patient with severe Crohn's disease. Dig Dis Sci 1997; 42: 823.

11. Jarrett P, Dufill M, Oakley A, Smith A. Pellagra, azathioprine and inflammatory bowel disease. Clin Exp Dermatology 1997; 22: 44-45.

12. Roubenoff R, Hoyt J, Petri M, et al. Effects of antiinflammatory immunosuppressive drugs on pregnancy and fertility. Semin Arthritis Rheum 1988; 18: 88-110.

13. Connell WR. Safety of drug therapy for inflammatory bowel disease in pregnant and nursing women. Inflammatory Bowel Dis 1996; 2: 33-47.

14. Alstead EM, Ritchie JK, Lennard-Jones JE, Farthing MJG, Clark ML. Safety of azathioprine in pregnancy in inflammatory bowel disease. Gastroentero logy 1990; 99: 443-446.

15. Kinlen LJ. Incidence of cancer in rheumatoid arthritis and other disorders after immunosuppressive treatment. Am J Med 1985; 78 : 44-49.

16. Connell WR, Kamm MA, Dickson M, Balkwill AM, Ritchie JK, LennardJones JE. Long-term neoplasia risk after azathioprine treatment in inflammatory bowel disease. Lancet 1994; 343: 1249-1251.

ACKNOWLEDGEMENT. The author thanks Professor J.H. Paul Wilson for his advice during the preparation of this paper.

\section{Received 27 February 1998; accepted 9 March 1998}




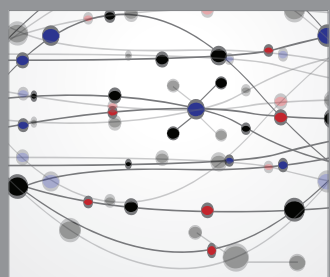

The Scientific World Journal
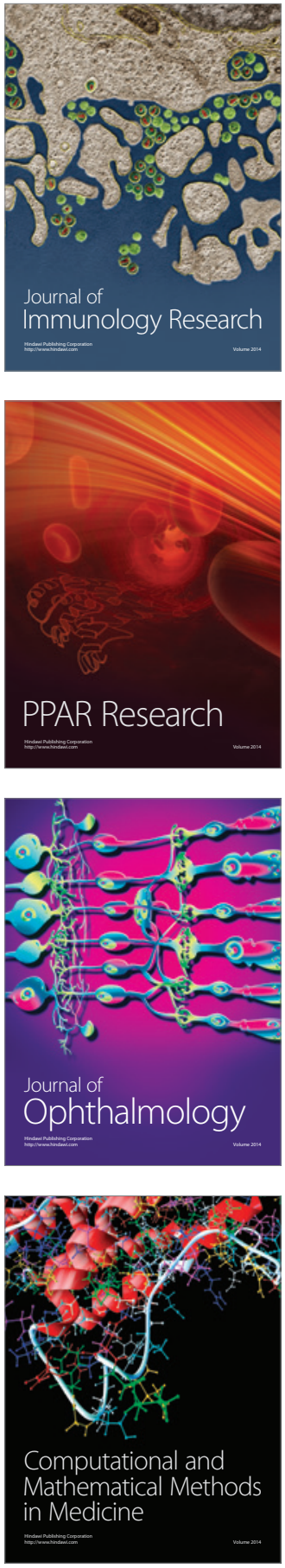

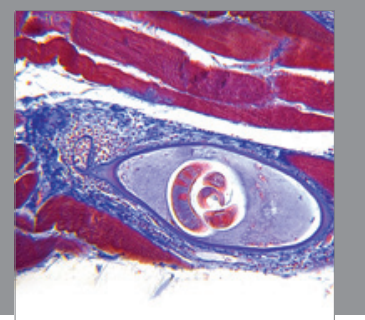

Gastroenterology

Research and Practice
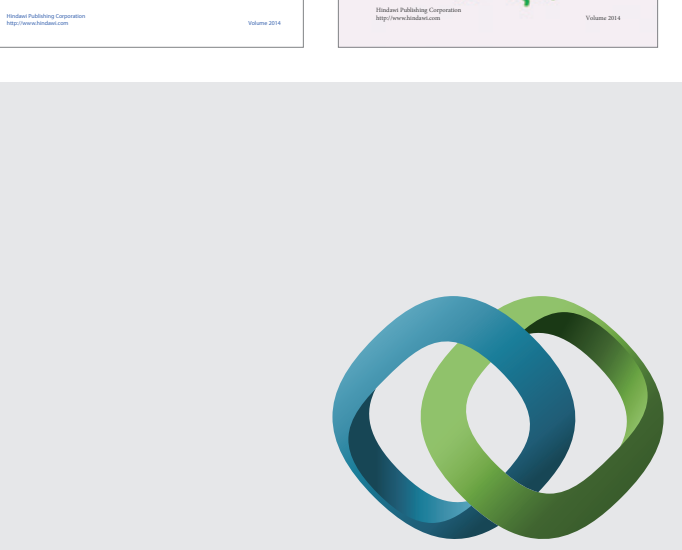

\section{Hindawi}

Submit your manuscripts at

http://www.hindawi.com
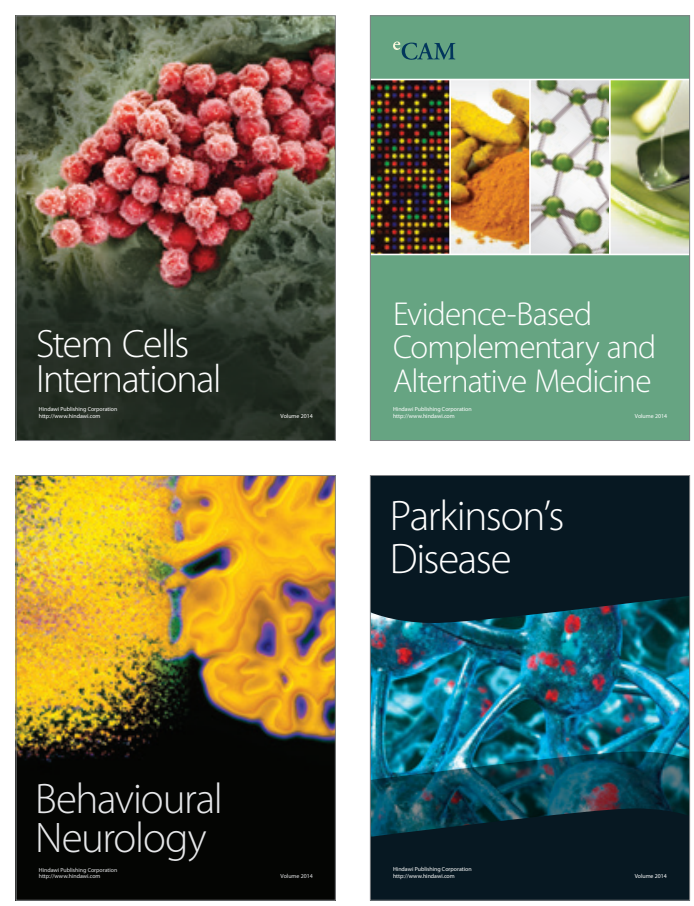

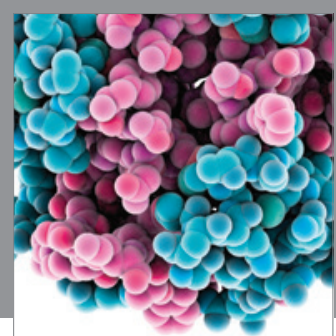

Journal of
Diabetes Research

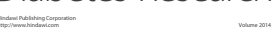

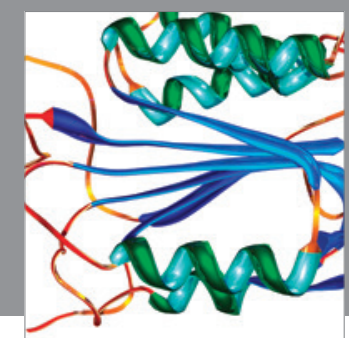

Disease Markers
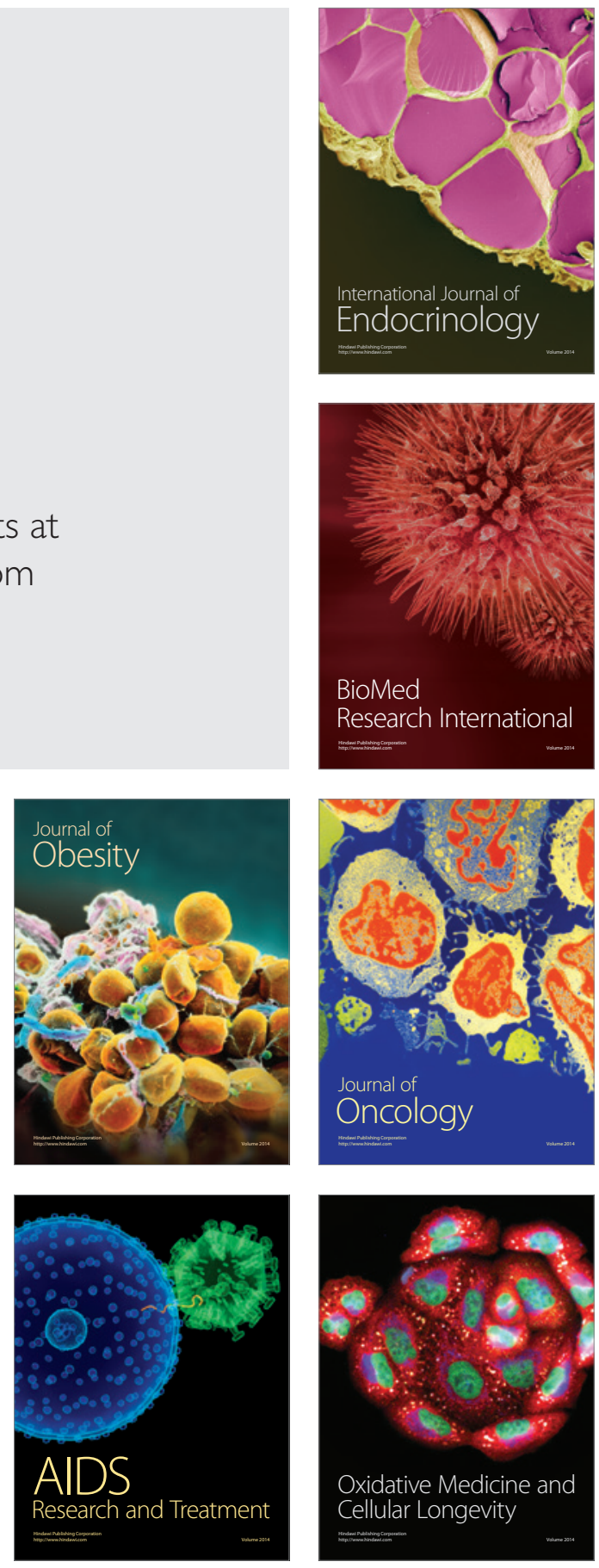